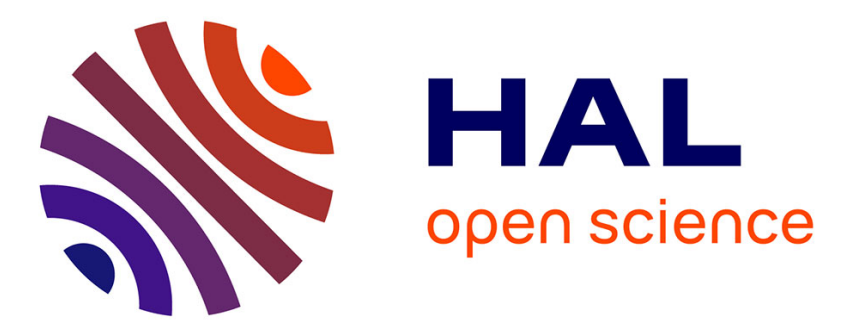

\title{
Local and global spatio-temporal entropy indices based on distance- ratios and co-occurrences distributions
}

\author{
Didier G. Leibovici, Christophe Claramunt, Damien Le Guyader, David
}

Brosset

\section{- To cite this version:}

Didier G. Leibovici, Christophe Claramunt, Damien Le Guyader, David Brosset. Local and global spatio-temporal entropy indices based on distance- ratios and co-occurrences distributions. International Journal of Geographical Information Science, 2014, 28 (5), pp.1061-1084. 10.1080/13658816.2013.871284 . hal-01208089

\section{HAL Id: hal-01208089 \\ https://hal.science/hal-01208089}

Submitted on 14 Feb 2017

HAL is a multi-disciplinary open access archive for the deposit and dissemination of scientific research documents, whether they are published or not. The documents may come from teaching and research institutions in France or abroad, or from public or private research centers.
L'archive ouverte pluridisciplinaire HAL, est destinée au dépôt et à la diffusion de documents scientifiques de niveau recherche, publiés ou non, émanant des établissements d'enseignement et de recherche français ou étrangers, des laboratoires publics ou privés. 


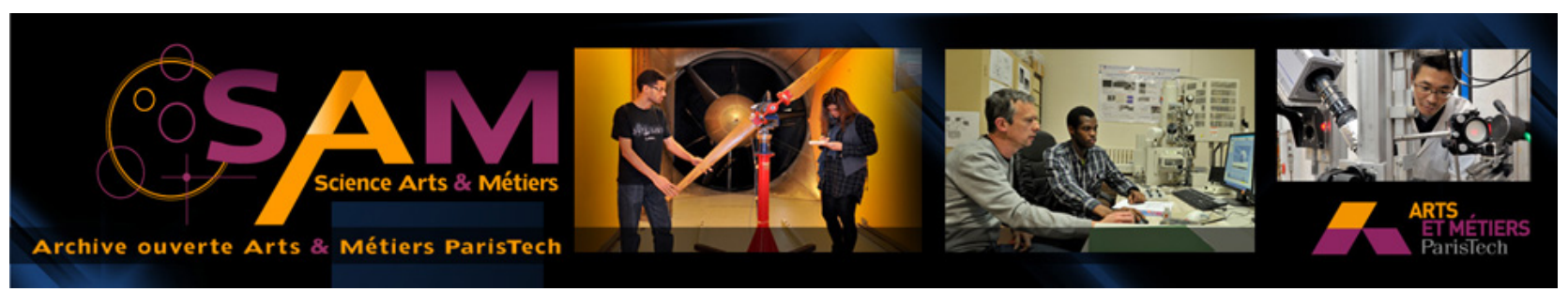

\section{Science Arts \& Métiers (SAM)}

is an open access repository that collects the work of Arts et Métiers ParisTech researchers and makes it freely available over the web where possible.

This is an author-deposited version published in: http://sam.ensam.eu

Handle ID: .http://hdl.handle.net/10985/10299

\section{To cite this version :}

Didier G. LEIBOVICI, Christophe CLARAMUNT, Damien LE GUYADER, David BROSSET - Local and global spatio-temporal entropy indices based on distance- ratios and co-occurrences distributions - International Journal of Geographical Information Science - Vol. 28, n5, p.1061$1084-2014$ 


\title{
Local and global spatio-temporal entropy indices based on distance-ratios and co-occurrences distributions
}

\author{
Didier G. Leibovici ${ }^{\mathrm{a} *}$, Christophe Claramunt ${ }^{\mathrm{b}}$, Damien Le Guyader ${ }^{\mathrm{c}}$ and David Brosset ${ }^{\mathrm{b}}$ \\ ${ }^{a}$ Nottingham Geospatial Institute, University of Nottingham, Nottingham, UK; ${ }^{b}$ Naval Academy \\ Research Institute, Brest Naval, France; ${ }^{c}$ University of Bretagne Occidentale, UMR LETG, Géomer, \\ IUEM, Plouzané, France
}

\begin{abstract}
When it comes to characterize the distribution of 'things' observed spatially and identified by their geometries and attributes, the Shannon entropy has been widely used in different domains such as ecology, regional sciences, epidemiology and image analysis. In particular, recent research has taken into account the spatial patterns derived from topological and metric properties in order to propose extensions to the measure of entropy. Based on two different approaches using either distance-ratios or co-occurrences of observed classes, the research developed in this paper introduces several new indices and explores their extensions to the spatio-temporal domains which are derived whilst investigating further their application as global and local indices. Using a multiplicative space-time integration approach either at a macro or micro-level, the approach leads to a series of spatio-temporal entropy indices including from combining co-occurrence and distances-ratios approaches. The framework developed is complementary to the spatio-temporal clustering problem, introducing a more spatial and spatio-temporal structuring perspective using several indices characterizing the distribution of several class instances in space and time. The whole approach is first illustrated on simulated data evolutions of three classes over seven time stamps. Preliminary results are discussed for a study of conflicting maritime activities in the Bay of Brest where the objective is to explore the spatio-temporal patterns exhibited by a categorical variable with six classes, each representing a conflict between two maritime activities.
\end{abstract}

Keywords: information theory; entropy; spatio-temporal entropy; co-occurrence data; nearest neighbor; spatial structuring; point pattern analysis

\section{Introduction}

The concept and measure of entropy as initially introduced by Shannon in his seminal theory of information has been long applied to many scientific domains to qualify the distribution of 'things' in space (Shannon 1948, Shannon and Weaver 1949). Shannon's entropy is a mathematical index that measures diversity in categorical data. It is more formally given by

$$
H(C)=-K \sum_{c} p_{c} \log \left(p_{c}\right)
$$

where $p_{c}$ is defined as the proportion of entities of the class $c$ of the categorical variable $C$ with $|C|$ classes ( $C$ being one attribute of the objects observed) and $K$ is a positive

*Corresponding author. Email: didier.leibovici@nottingham.ac.uk 
constant. The entropy $H$ is a positive value, and it is bounded by the unit interval when $K$ is chosen as the inverse of the maximum entropy among discrete distributions with the same number of classes. This maximum value, $\log (|C|)$, is reached when the distribution of classes is uniform $\left(\forall c, p_{c}=n_{c} / N=1 /|C|\right)$, which increases with the number of classes, so that $H$ with this normalizing factor describes the structuring of the distribution as departing from the uniform distribution.

Since the seminal contribution of Shannon the measure of entropy has been widely applied in various domains including for spatial and geographical data, such as in regional sciences, image analysis, ecology and social sciences, see some review in Li and Huang (2002), Claramunt (2005), Leibovici (2009) and Batty (2010). In ecological and environmental studies (Margalef 1958, Menhinick 1964, McIntosh 1967, Hurlbert 1971, Gonzalez and Chaneton 2002), directly applied to the spatial data, the measure of entropy has been used to evaluate the fragmentation and spatial heterogeneity of geographical phenomena (O’Neill et al. 1988, McGarigal and Marks 1994). One of the first attempts to integrate some specific spatial properties in the measure of entropy was suggested by $\mathrm{Li}$ and Reynolds (1993). The main idea behind this was to quantify a measure of contagion and to which extent regions of a given class are adjacent to regions of another class, allowing to evaluate the degrees of juxtaposition and aggregation of the categorical data. Other measures such as dominance have been also suggested (Riitters et al. 1996). In fact, contagion is inversely correlated to diversity. For a given number of classes, the contagion is minimum when all classes are evenly distributed and equally adjacent to each other. As such the measure is essentially expressing a local configuration tendency, the overall spatial structure of the categorical data considered deriving from the accumulation of local information. In regional science where areal data with weights has to be taken into account, a density-based measure of entropy has been applied to the study of a probability distribution over a progressive distance from a given location (Batty 1974), but still, the relative spatial distribution of the classes is not taken into account. With similar approaches to regional science, segregation analysis coming mainly from social science has been using entropy measures for spatial data (e.g., Wong 2002, Reardon and O'Sullivan 2004). Besides using density-based entropy, spatiality in the entropy measure is taken into account with local measures derived using neighborhoods densities of each category (e.g., social groups) using a spatial proximity matrix, see also Karlstrom and Ceccato (2002). This chosen spatial proximity matrix is also used to derive exposure indices between two groups or one to the others from a correlation like formula in similar way to a local Moran's Index (Anselin 1995). In this approach each category of the categorical variable studied is reified as one variable observed on areal data (proportion of each category in each unit), therefore usually looking at the spatial structuring of one category or spatial correlation between two groups like in hot-spot maps (see also Section 3).

The main objective of this paper is to be able to relate the spatial or spatio-temporal pattern of several categories together in one single index as a global value or as a spatial map expressing local values. The focus of the paper is limited to explore how the concept and previous measures of entropy can fulfill this objective.

In a previous work, a measure of spatial entropy has been introduced to take into account the role of spatial distances between classes when applying a measure of entropy (Claramunt 2005). The idea behind this notion is to consider the primal role of distance toward the spatial structure of a given system. The principle of this spatial entropy is that this measure should augment when distance between dissimilar entities decreases, as well as the entropy should augment when the distance between similar entities increases. As such this measure gives an overall and global index of the relative distribution in space of 
the classes. On the other hand, local arrangements as well as cross-relations between the classes are not directly evaluated. With this aim a related parallel work on local interactions between co-occurrences of categorical data has been studied and formalized into another spatial measure of entropy (Leibovici 2009; Leibovici et al. 2011b). The main idea of the latter indices, suggested as generalizing contagion indices, is to consider as spatial information the distribution of co-occurrences between two or more observations with varying vicinities for the exploration of spatial patterns at different scales. It appears that these two approaches take different topological points of view when extending the measure of entropy to spatial observations: local versus global, co-occurrence versus relative spatial distribution. The principle of the co-occurrence-based entropy is very similar to the symbolic entropy framework developed in regional science by Lopez et al. (2010) and Matilla-García et al. (2012), nonetheless the occurrence of a symbol being defined as matching the nearest neighbors pattern of categories may be quite restrictive and strict for spatial dependence whereas co-occurrence as defined in Leibovici (2009) brings more flexibility (see Section 2.1).

Whilst the aim of the paper is to extend these approaches under the interrelations of the spatial and temporal dimensions, the research developed in this paper explores the complementarity of these two families of indices, and evaluates to which degree the combination of the two might enrich the way a given phenomenon distributed in space and time can be analyzed in order to apprehend its spatio-temporal structuring.

Spatial structuring analysis can be seen as the alter ego of spatial clustering analysis, where in the latter the focus is more on locating and delineating clusters often from testing a hypothesis referring to a null distributional assumption. The semantic behind the wording structuring is more related to pattern, modeling, association and to the apprehending of the spatial observations as a whole from which the existence of clusters is one aspect among other structuring characteristics. Spatio-temporal clustering from a structuring point of view comes from disciplines such as ecology with criteria like diversity, richness, patchiness and associations whereas detecting structures by locating clusters is often linked to outbreak detection as in epidemiology. Local and global approaches have proven to be useful in spatial analysis and are also at the source of a plethora of spatial and spatio-temporal methods to do with structuring and tests for clustering (Haining 2003, Tango 2010, Bivand et al. 2013).

Concepts such as composition and configuration echoing global and local properties (Li and Reynolds 1993, Karlström and Ceccato 2002, Boots 2003, Okabe et al. 2010) are at the root of a proper description of the spatial distribution of a categorical variable; these terms are directly linked to occurrences and co-occurrences. Global configuration can be also intrinsically expressed by distances between the observations of the classes involved. Ripley's $K$ function (Ripley 1977), seen as the ratio of the expected number of further points from a random point location for a given spatial lag (distance) to the local intensity, is a global statistic that describes the structuring which in fact expresses the natural dependency between composition and configuration when studying the second order moment of a spatial point process. The shape of the curve of the observed values for $K$ at different spatial lags and its comparison to a theoretical curve obtained under particular hypothesis such as complete spatial randomness (CSR) helps to describe the global pattern and test it (e.g., Bivand et al. 2013). Cross- $K$ statistics extend the approach to two sets of points with a categorical variable identifying the points. Nonetheless with more than two categories (classes) using cross- $K$ functions for all pairs of categories (or pooling all other categories against one) may be limited when describing the global spatial structuring. Higher-order co-occurrences have been proven to be useful at describing more complex 
associations including using extensions of Ripley's $K$ (Leibovici et al. 2011b). Unlike most of the statistics based on second order moments the entropy allows to grasp all categories at once. The entropy indices introduced aim at enriching the conventional measures of entropy as identified by the information theory by taking into account the spatial and temporal dimensions structuring the information. Nonetheless our approach may also benefit the research into spatio-temporal clustering within an outbreak detection sense as entropy-based testing have also been proven to be as powerful as classical approaches for spatial dependence (Matilla-García et al. 2012).

The rest of the paper is organized as follows. Section 2 introduces the measures of distance-based and co-occurrence-based spatial entropies, while Section 3 studies the local and global aspects of the indices. Section 4 extends our approach to the temporal dimension and discusses the application potential of the whole framework. Whilst previous sections were illustrated with a simulated dataset, Section 5 describes preliminary results obtained for a study of conflicts of maritime activities in the Bay of Brest (France) in 2009. Finally the conclusion summarizes the contribution and outlines further work.

\section{Distance-based and co-occurrence-based spatial entropy}

Along the paper the principles of the various spatial and spatio-temporal entropy indices are illustrated using simulated data with 3 classes of 60 points evolving from time 1 to time 6. The purpose of this simulation is to generate and control the spatio-temporal structure of the data using a known evolution process with simple structuring for each class. Evaluating to which degree the methodology described in the paper could pick up the existence of the deterministic part of the evolution process is beyond the scope of the paper. Figure 1 shows these three classes generated with different initial distributions and evolutions. At time 1 the classes were generated on a unit square window as: + with random uniform coordinates $x$ and $y, \mathbf{o}$ idem with a tendency to cluster in the upper right using a density proportional to $x y$ and $\Delta$ with random uniform coordinates with a tendency to cluster in the bottom right corner using a density proportional to $x^{2}(1-y)^{2}$. At each
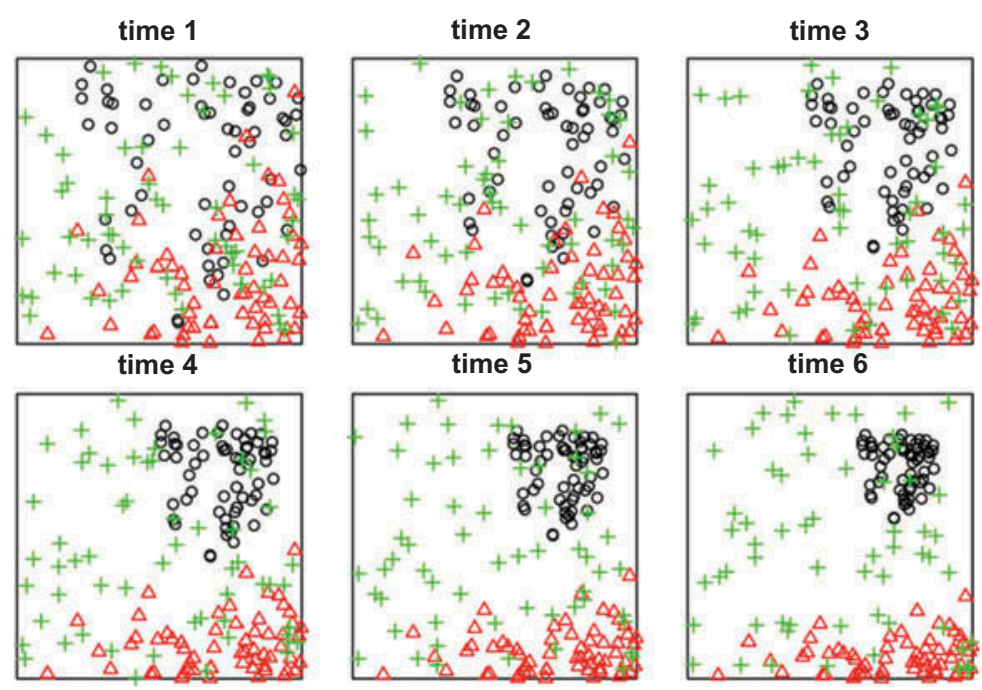

Figure 1. Spatio-temporal point pattern simulated with three classes. 
new time step the points of class + have Gaussian shifts, the points of class o move linearly (coefficient 0.2 ) toward the point in the top right corner of coordinates $(0.8,0.8)$, and the points of class $\Delta$ move toward the horizontal axe proportionally to the vertical coordinate (with coefficient 0.2 ).

For each of these six marked point patterns the normalized Shannon entropy (1) of the observed distribution is equal to 1 , as the distribution of marks (classes) is uniform. Nonetheless, the observations of these classes, except the points of class + , clearly exhibit a spatial organization, a pattern which becomes more and more evident with time. The research aim of the paper is to develop appropriate spatial and spatio-temporal entropy statistic being able to detect the existence of this structuring that emerge from these evolution patterns.

As being not just a distribution over a set of spatial units, a spatial distribution should reflect the information about the contiguity of observations with dependencies on attribute values. For example, in Figure 1 even if the count of occurrences of observations is the same at time 1 and time 6 , a spatial entropy at time 6 should be smaller as points are more organized for the classes o and $\Delta$. Similarly, permuting randomly the pixels of an image should not either give the same entropy. Therefore using solely the frequency distribution of the classes in the entropy index cannot reflect the structures that emerge in space and time. In order to take into account the spatial or spatio-temporal dimensions within an entropy index, the approach developed aims at integrating two topological and metric criteria in the definition of the entropy: distances between and within classes, and cooccurrences of observations of the same class or of different classes.

\subsection{A co-occurrence-based spatial entropy}

The proximity of the occurrences for a particular class is crucial when assessing the spatial distribution of a categorical variable. The concept of co-occurrence, that is, a set of observations located within a given spatial zone, is therefore a characteristic to take into account when describing the spatial distribution. In order to take into account the spatial patterns that emerge from several classes, a measure of entropy can be defined using the co-occurrences distribution (Leibovici 2009; Leibovici et al. 2011b). This is devised as a generalization of the adjacency index (O’Neill et al. 1988, Li and Reynolds 1993). A cooccurrence distribution is obtained, in a practical approach, by counting the number of collocated sets of $k$ observations ( $k$ is called the order of co-occurrence), a collocation taking place when the distance between any two observations of the set is less than a chosen threshold $d$, the collocation distance. Depending on the attributes of the observations to record for the co-occurrences, the co-occurrence distribution is finally characterized. The $k$-spatial entropy of a categorical variable $C$ with a total of $|C|$ classes observed spatially is a total of $N$ points is defined as

$$
H_{k S}(C, d)=-1 / \log \left(\left|C_{o o}\right|\right) \Sigma_{c_{o o}} p_{c_{o o}, d} \log \left(p_{c_{o o}, d}\right)
$$

where $p_{c o o}, d$ is the multinomial distribution associated to the multi-entry table of counts of co-occurrences of order $k$ at collocation distance $d$; the multi-index $c_{o o}$ relates to the chosen attributes to record, associated to the chosen categorical variable(s). Therefore, $\left|C_{o o}\right|$, the number of different values that the multi-index $c_{o o}$ for the categorical variable $C$ can take, is the number of co-occurrence classes. The normalizing value of $\log \left(\left|C_{o o}\right|\right)$ corresponds to the maximum value of the Shannon entropy for a uniform distribution of these classes, then $0 \leq H_{k S} \leq 1$. With a multi-index $c_{o o}$ chosen as a 
repetition of a class according to the order $k$, e.g., $p_{c o o}, d=p_{c c c}, d$ when $k=3$ for any class $c$, this entropy measure is termed self- $k$-spatial entropy $H_{k S}^{s}$ :

$$
H_{k S}^{s}(C, d)=-1 / \log (|C|) \sum_{i} p_{c c c \ldots c, d} \log \left(p_{c c c \ldots c, d}\right)
$$

The distribution used in the self- $k$-spatial entropy comes from keeping only the hyperdiagonal of the multi-entry table of co-occurrences counts that would be used for the $k$-spatial entropy: in Equation (3) $\left|C_{o o}\right|=|C|$, in Equation (2) $\left|C_{o o}\right|=|C| *|C| *|C|$ with $k=3$. Therefore, $H_{k S}^{s}$ the self- $k$-spatial entropy appears as a multinomial-univariate index version of $H_{k S}$, the $k$-spatial entropy which is multinomial-multivariate. While the classical entropy is based on the distribution of occurrences of the classes, the self- $k$-spatial entropy takes into account the distribution of co-occurrences of these classes.

In the rest of the paper the co-occurrence-based entropy uses only the self- $k$-spatial entropy as in first instance we are interested in trends emerging from the set of classes but not in the associations of the classes. This choice is driven by a more direct analogy then, for the two approaches of co-occurrences and distance-ratios, of the way the topology of the classes is taken into account, thus allowing potential integration of the two approaches. The multivariate aspect of the $k$-spatial entropy is also more demanding on sample sizes (Leibovici et al. 2011b).

In Figure 2 the choice of a range of collocation distances illustrates the sensitivity and potential scale analysis provided by the self- $k$-spatial entropy. This shows that, independently of the evolution, a collocation distance too small may induce a false impression of pattern structure, while a too large one can also be not appropriate to identify the emerging spatial structure. Regarding the patterns detected, at a local scale the entropy values decrease with time while at a higher scale from a collocation distance $d \geq 0.4$, the entropy becomes relatively stable after time 4 . That distance $d$ where the observed entropy changes rapidly (between 0.0 and 0.4 here) can be considered as an important threshold for the analysis of the scale of the studied data. Moreover, using a range of collocation

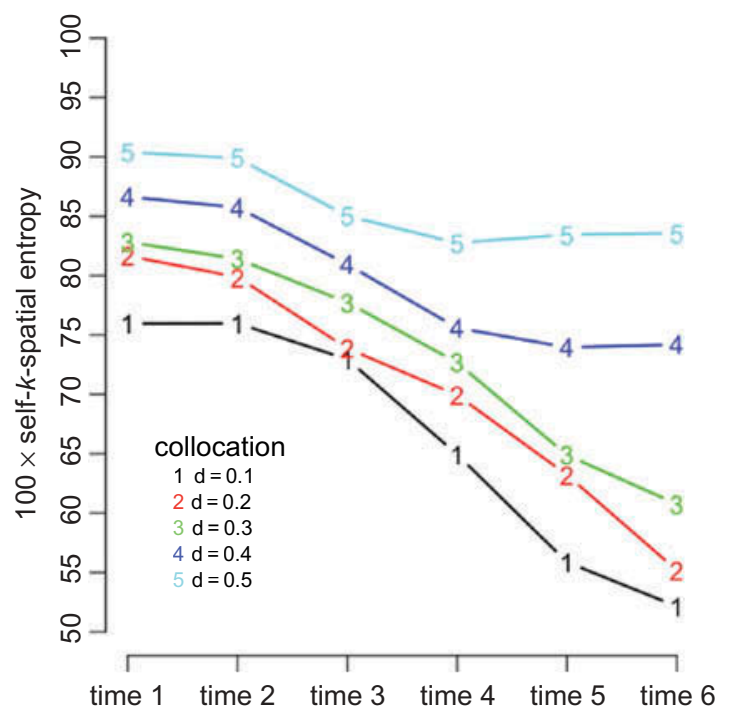

Figure 2. Evolution over time of the self- $k$-spatial entropy with different collocation distances. 
distances allows consistent pattern detection, here identified from the simulated data. The plateau of entropy values, observed from $d \geq 0.4$ in Figure 2 illustrates the 'maximum' scale structure which can be detected but up to $d=0.3$ denser clustering structures are identified up to the end of the evolution.

\subsection{Discriminant-ratio-based spatial entropy}

The distances between the observed classes can also reveal the 'spatial correlation' of the classes. Intuitively, if the observations for a particular class tend to cluster relatively to the other classes, the distribution of distances between pairs of observations from that class should be narrower and shifted toward small values than the distribution of pairs of observations with one observation not in that class. For a categorical variable $C$ with a distribution of observations $p_{c}=n_{c} / N$ ( $n_{c}$ being the number of observations of the class c and $N$ the total number of observations), a measure of spatial diversity has been introduced using a ratio distances between pairs of observations for similar and different classes (Claramunt 2005):

$$
H_{s}(C)=-\Sigma_{c} \frac{d_{c}^{\text {int }}}{d_{c}^{\text {ext }}} p_{c} \log \left(p_{c}\right)
$$

where $d_{c}^{\text {int }}=1 /\left(2 * n_{\{c, c\}}\right) \sum_{r \in c, r^{\prime} \in c} d_{r r^{\prime}}$ is the average distance between a pair of observations belonging to the same class $c$, called intra-distance; $d_{c}^{\text {ext }}=1 / n_{\left\{c, c^{\prime} \neq c\right\}} \sum_{r \in c, r^{\prime} \in c^{\prime}} d_{r r^{\prime}}$ is the average distance between a pair of observations, where only one belongs to the class $c$, called the extra-distance (the number of pairs are, respectively, $n_{\{c, c\}}=n_{c}\left(n_{c}-1\right) / 2$ and $n_{\left\{c, c^{\prime} \neq c\right\}}=n_{c}\left(N-n_{c}\right)$ ). The ratio of these distances appears as a discriminant statistic between classes with compact observations relatively to the other classes, giving a ratio smaller than 1 , and classes more dispersed relatively to the others giving a ratio greater than 1 .

Taking the inverse of this ratio one gets, after normalization, a probability with a similar aim as with the co-occurrence distribution (high probability for compact clustered observations of a given class):

$$
H_{d S}(C)=-1 / \log (|C|) \Sigma_{c} d_{c}^{\rho} \log \left(d_{c}^{\rho}\right)
$$

where $d_{c}^{\rho}=\left(\frac{d_{c}^{\text {ext }}}{d_{c}^{\text {dint }}}\right) /\left(\sum_{c} \frac{d_{c}^{\text {ext }}}{d_{c}^{\text {int }}}\right)$ is the normalized ratio of the distances. $H_{d S}$ is easier to interpret than $H_{S}$ but one loses the relative distribution of observations of the classes, which can be re-integrated by doing the symmetric of Equation (4):

$$
H_{d S}^{p}(C)=-|C| / \log (|C|) \Sigma_{c} p_{c} d_{c}^{\rho} \log \left(d_{c}^{\rho}\right)
$$

Or

$$
H_{p d S}(C)=-|C| / \log \left(|C|^{2}\right) \Sigma_{c} p_{c} d_{c}^{\rho} \log \left(p_{c} d_{c}^{\rho}\right)
$$

which can be seen as the normalized entropy for the diagonal of the joint distribution of the observations of the classes and their compactness under the hypothesis of independence. 


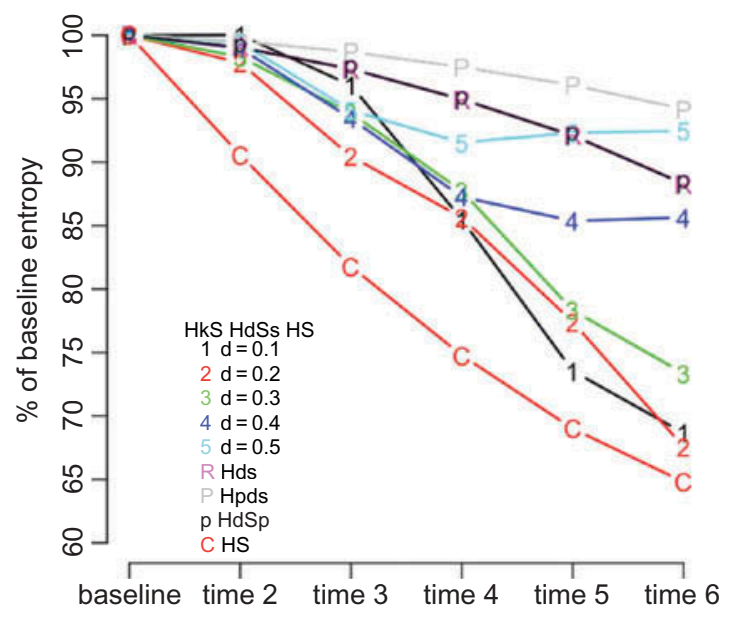

Figure 3. Comparison of the evolution of the ratio to baseline of the entropy indices: HkS is the self- $k$-spatial entropy (3), HdSs (HdS, HpdS, HdSp) and HS are the distance-based indices in Equations (5), (7), (6) and (4), respectively.

When comparing the values of the different entropy indices so far introduced, and according to their evolution for this particular simulated dataset, the discriminant-ratiobased entropy $H_{S}$ appears to be the most responsive to the spatial structuring and its evolution, nonetheless in a 'linear' way relatively to the evolution. The self- $k$-spatial entropy index $H_{k S}^{s}$, at medium collocation distances, performs well whilst being more able to show the acceleration of the spatial structuring. The new indices based on the discriminant-ratio, $H_{d S}, H_{p d S}$ and $H_{d S}^{p}$ are less able to capture the dramatic changes in the evolution. One must notice that because of the uniform distribution of the classes (globally), the 'entropy part' of the index $H_{S}$ is the same for all the classes, so does not influence the changes (Figure 3). The index works only on the discriminant-ratios in this case.

\section{Local and global indices}

The spatial entropy indices developed in the previous section are global statistics that should be completed by local indices that can identify where (or when or both) the spatial or time structure is the most determinant. Particularly low and high values along with their autocorrelation and potential grouping may be then looked for and a post-hoc testing analysis may be then applied to estimate their significance (Leibovici et al. 2011a). Complementing global statistics by their local equivalent has been studied by spatial correlation measures in geostatistics and spatial analysis with applications in geographical information science (GIS). One can mention LISA statistics (Anselin 1995) with the Moran's Index, or the Getis-Ord statistic (Getis and Ord 1992), which are frequently used for continuous variables represented by 'hot-spot' maps, thus could be used for post-hoc analysis. When considering categorical data, scan statistics have been also applied using for example SaTScan and GAM methods (Openshaw et al. 1987, Kulldorff and Nagarwalla 1995), and where a window around a set of observations is applied using a maximum likelihood ratio statistic that compares inner and outer windows: the set of observations form a cluster if the statistic is significant enough. As described in Leibovici et al. (2011a), a SaTScan method for 
multinomial data has been developed by Jung et al. (2010) with as alternative hypothesis that the proportions for at least one of the categories show different values between the inner and outer windows of the scan, so not necessarily pointing out a particular distributional structure of the categories in the inner window.

\subsection{Local contributions to global statistics}

While LISA's statistic properties assure that the sum of the values is proportional to the global statistic, this is difficult to achieve here because of the use of logarithms in the indices introduced in the previous sections. Nonetheless, co-occurrences and discriminant distances-ratios, accumulated from local observations, can provide meaningful local evaluations that can also give some insights regarding the global distribution. For the discriminant-ratio-based entropy the ratio of the averages of the distances inter and extra can give a local evaluation:

$$
\frac{d_{i}^{\text {int }}}{d_{i}^{\text {ext }}}\left(V_{i}\right)=\left(n_{\text {ext }}\left(V_{i}\right) / n_{\text {int }}\left(V_{i}\right)\right) \Sigma_{r \in c(i) \cap V_{i}} d_{r i} / \Sigma_{r \notin c(i), r \in V_{i}} d_{r i}
$$

for a neighborhood $V_{i}$ for each observation $i,\left(n_{\text {ext }}\left(V_{i}\right)\right.$ and $n_{\text {int }}\left(V_{i}\right)$ being the count of observations for each sum). Equation (8) becomes (4) if the vicinity rule is, $V_{i}=V$, i.e., the whole study area. The neighborhood $V_{i}$ can be defined (a) from a zoning system (e.g., administrative or naturally defined units) where each $V_{i}$ matches one of the fixed units containing the observation $i$ or (b) from an algorithmic constraint where $V_{i}$ is a buffer around each observation $i$ using a buffer distance dependent or not from $i$ or (c) where $V_{i}$ is a varying buffer chosen according to sample size using nearest neighbors. Overall, the vicinity rule allows expressing a local characteristic at each observation.

In Figure 4 a map of the discriminant-ratios computed at each point using (8) shows the overall influence of the observations from all classes to a particular (localized) class element: a small value reveals a tendency to be in a cluster of the observed class. In Figure 4 the values get smaller with the evolution, and they are even smaller for the class o, confirming the spatial structure.

Results in Figure 5, with $V_{i}$ being not the whole space and constituting a local vicinity of the 90 nearest neighbors for each observation $i$, are comparable but with less evidence of clustering for less compact clusters, e.g., at time 4. This seems logical as the distances are then limited within the local neighborhood. Nonetheless local clustering for a nonglobally clustered class is better depicted than when using the whole space. This is somehow confirmed when comparing the time 6 between Figures 4 and 5, where the class + exhibits visually a zone in between the other two classes, seen as a structuring cluster by the lack of observations from other classes: small values in Figure 5 but not in Figure 4.

Regarding the self- $k$-spatial entropy, the local contributions to the global statistic emerge from the local co-occurrence counts for the observed class, i.e., co-occurrences with the current observation as illustrated in Figure 6. A high value means a tendency to be in a cluster of the observed class, and the emerging clusters are depicted by the evolutions of these local contributions.

In Figure 6 the neighborhood is defined by the collocation vicinity. Using a larger neighborhood $V_{i}$ allows to consider local evaluation or local constraints instead of a local 
time 1

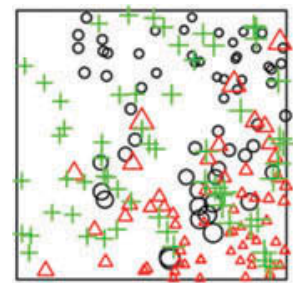

time 4

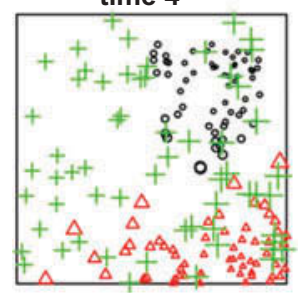

time 2

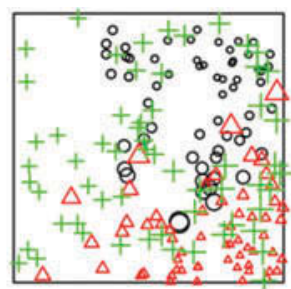

time 5

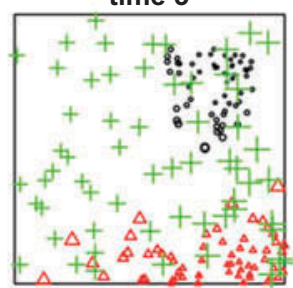

time 3

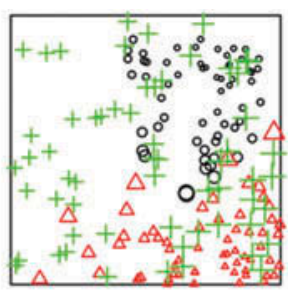

time 6

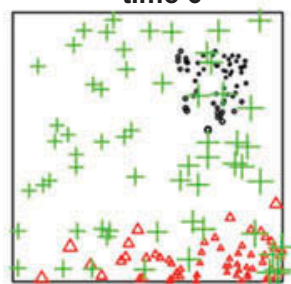

Figure 4. Map of the discriminant-ratios at each point Equation (8) with $V_{i}$ as the whole space. Sizes of labels are proportional to the values (longitudinally normalized).

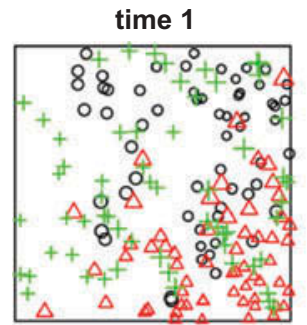

time 4

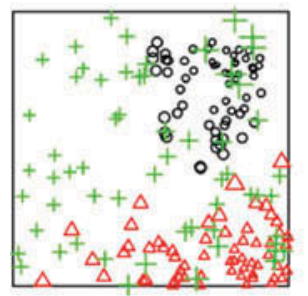

time 2

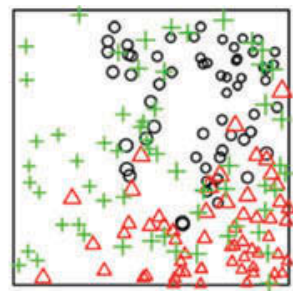

time 5

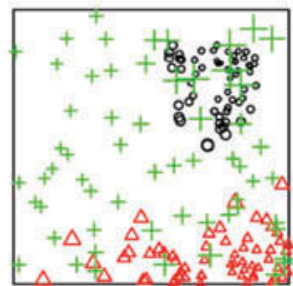

time 3

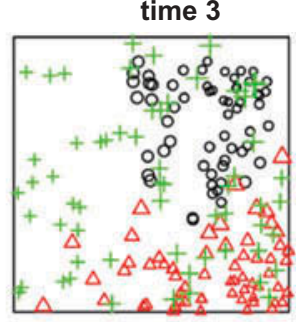

time 6

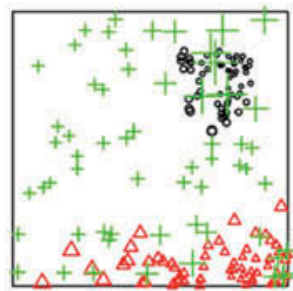

Figure 5. Map of the discriminant-ratios at each point Equation (8) with $V_{i}$ as a set of the 90 nearest neighbors. Sizes of labels are proportional to the values (longitudinally normalized).

contribution. In Figures 4 and 5 a scaling was applied so that the sum of all values over space and time are similar.

\subsection{Local evaluations and local constraints}

The introduction of a neighborhood parameter for the local contributions to the spatial entropy (using either a distance-ratio-based index or a $k$-co-occurrence one) allows a localization of the indices. Nonetheless, there is a difference between the two approaches: the co-occurrences-based indices being subject to the collocation distance and expressing 

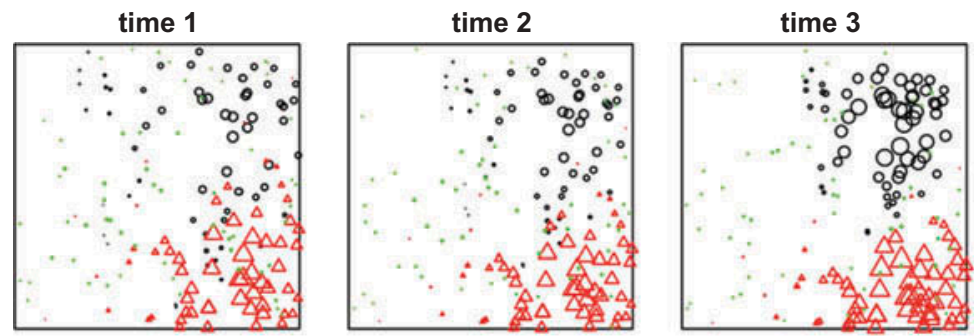

time 4

time 5
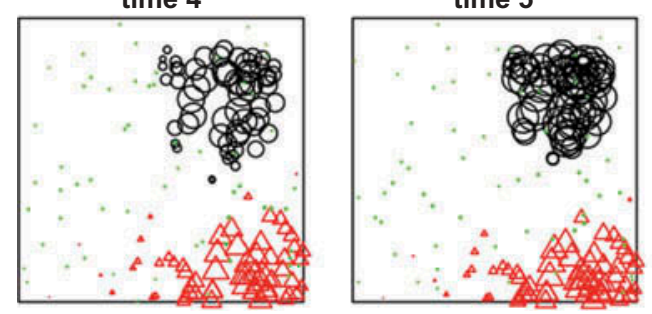

time 6

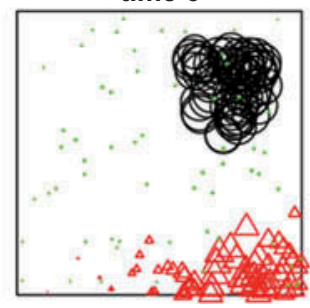

Figure 6. Map of the self- $k$-co-occurrence counts at each point with collocation distance $d=0.3$. Sizes of labels are proportional to the counts.

a scale effect are already locally focused. The co-occurrence distribution is an accumulation of counts but for the distance-based entropy indices, the discriminant-ratio is a global statistic as computed using all the observations available. Nonetheless, the distance-based entropy indices can be evaluated locally in the neighborhood of each observation or can be also evaluated globally but with a vicinity constraint when averaging the distances of pairs:

$$
\frac{d_{c}^{\text {int }}}{d_{c}^{\text {ext }}}(\mathcal{V})=\left(n_{\text {ext }}(\mathcal{V}) / n_{\text {int }}(\mathcal{V})\right) \Sigma_{r} \Sigma_{r^{\prime} \in c(r) \cap V_{r}} d_{r r^{\prime}} / \Sigma_{r} \sum_{r^{\prime} \notin c(r), r^{\prime} \in V_{r}} d_{r r^{\prime}}
$$

then leading to new versions of the discriminant-based spatial entropy indices of the Equations ((4)-(7)): $H_{S}(C, \mathcal{V}), H_{d S}(C, \mathcal{V}), H_{d S}^{p}(C, \mathcal{V}), H_{p d S}(C, \mathcal{V})$, where the vicinity rule leading to each $V_{r}$ is expressed by $\mathcal{V}$. The constraint expressed by the vicinity rule $\mathcal{V}$ is as described in the previous section, using either a fixed zoning constraint (the sets of all the neighborhoods of each observation defined by a buffer distance) or the number of nearest neighbors. In Figure 7, 90 nearest neighbors were used; the self- $k$-spatial entropy results from Figure 3 are repeated as a benchmark. In comparison to Figure 3 the index $H_{S}$ is discriminating less with the 90 neighbors constraint than without, but is still within the best results obtained for the self- $k$-spatial entropy with collocation distances between $0.1-$ 0.3 . The other distance-based entropy indices do not capture the clustering evolution of these three classes.

In Figure 8, the effect of different neighborhood sizes is analyzed on the indices from Equations (5) and (4), $H_{d S}(C, \mathcal{V}), H_{S}(C, \mathcal{V})$, respectively, and updated with the neighborhood constraint (9). The general emerging pattern is that the higher the numbers of neighbors, the more the indices capture the clustering evolution. Nonetheless, for the two indices 90 neighbors seems to be a tipping point from where the indices get their 'power', this is clearly visible for $H_{S}(C, \mathcal{V})$ bottom of Figure 8 but also apparent on the less powerful index $H_{d S}(C, \mathcal{V})$. Interestingly, and relatively to the baseline (time 1), 


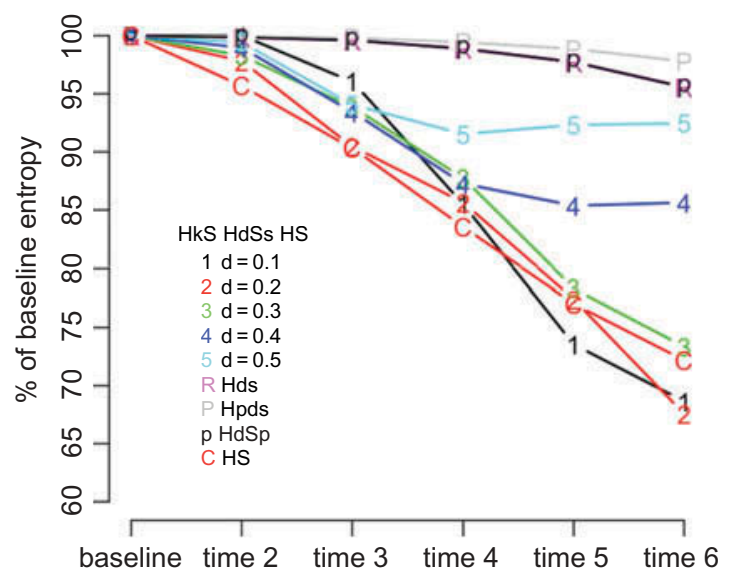

Figure 7. Comparison of the evolution of the ratio to baseline of the entropy indices for $H_{S}(C, \mathcal{V}), H_{d S}(C, \mathcal{V}), H_{d S}^{p}(C, \mathcal{V}), H_{p d S}(C, \mathcal{V})$ where the vicinity constraint is defined by 90 nearest neighbors at each location.
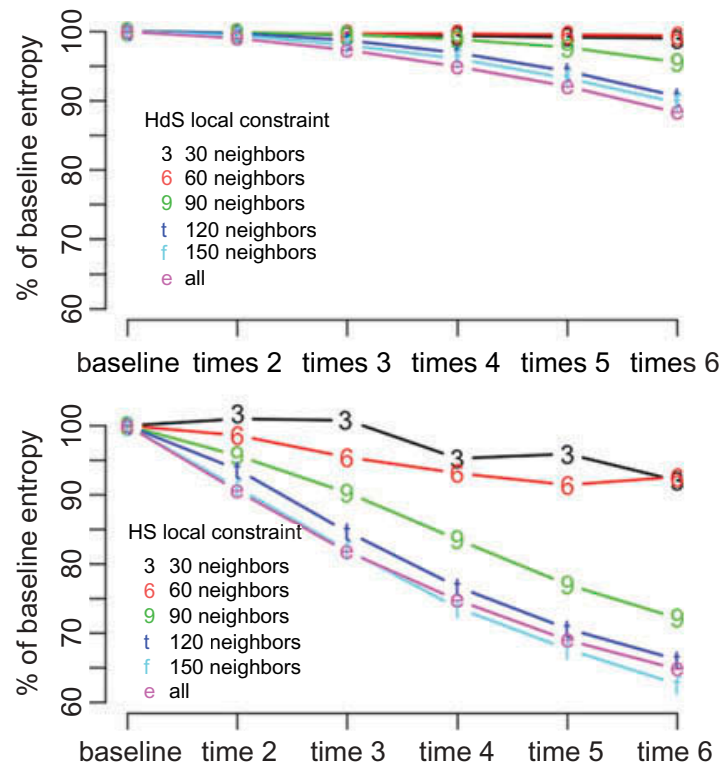

Figure 8. Effect of the size of the vicinity size of the vicinity constraint for $H_{d S}(C, \mathcal{V}), H_{S}(C, \mathcal{V})$.

$H_{S}(C, \mathcal{V})$ is less discriminant for the evolution with all the observations (no neighborhood constraint) than with the 150 nearest neighbors constraint.

The same vicinity rule can be also applied for local evaluations but the sample sizes have their importance. This approach has been used (Leibovici et al. 2011a) for a spatial scan statistic where the number of points considered for each local evaluation is given by the number of nearest neighbors within a 'hot-spot' mapping approach. It can be applied for both spatial entropy approaches taken here. 
In Figure 9, despite a decrease between time 1 and time 6, not much variation occurs: $H_{s}(C \mid \mathcal{V})$ shows more time variations while $H_{d s}(C \mid \mathcal{V})$ shows more spatial variations. Constraining too much the extra-distance $d_{c}^{\text {ext }}$ to local observations makes the distanceratio less discriminant. The self- $k$-spatial entropy evaluated locally works much better (Figure 10), but that was expected as the global statistic is based on aggregation of local counts of co-occurrences.

Co-occurrence-based indices being more locally dependent, the values displayed in Figure 10 highlight logically the local structuring and autocorrelation of this structuring. The local evaluations compare observed co-occurrences distributions to a local uniformity of co-occurrences, which can be seen as a local null hypothesis. The control within this statistic can be qualified as internal as varying the size of the scan window do not change the null hypothesis expected counterparts, unlike the principles used with SaTScan comparing inner and outer log likelihood (KullDorff and Nagarwalla 1995) or with

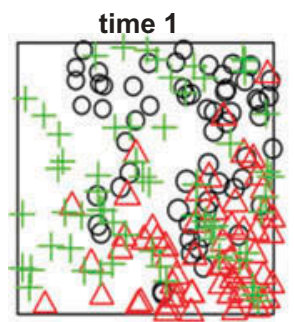

time 4

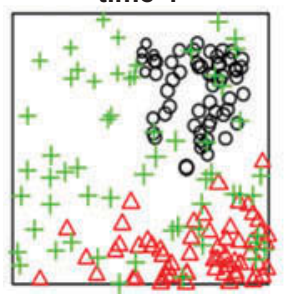

time 1

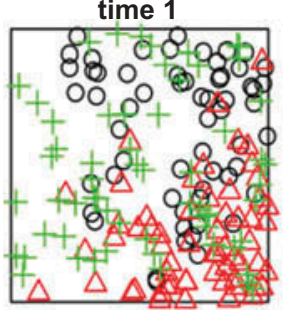

time 4

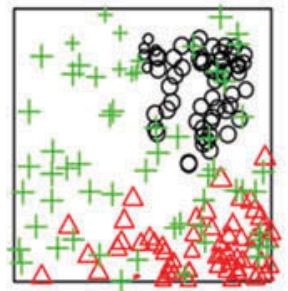

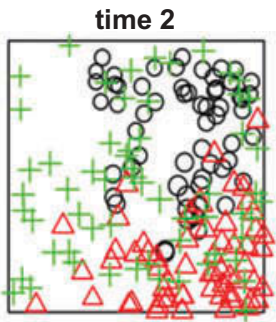

time 5
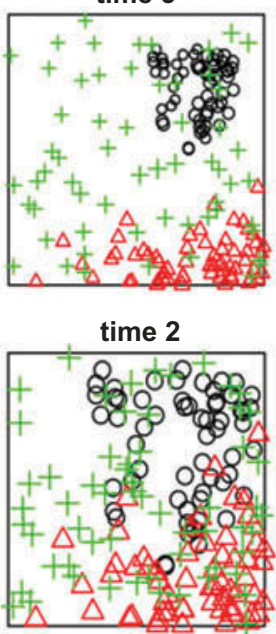

time 5

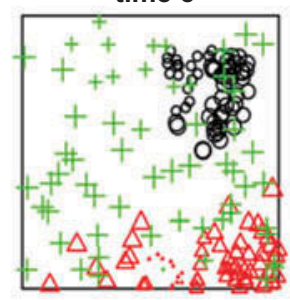

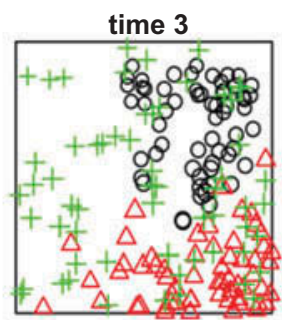

time 6

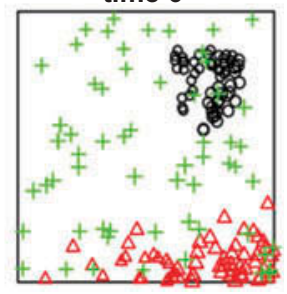

time 3

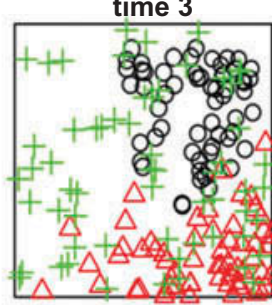

time 6

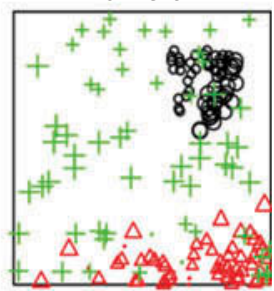

Figure 9. Local evaluations termed $H_{s}(C \mid \mathcal{V})$ (top) and $H_{d s}(C \mid \mathcal{V})$ (bottom) where $\mathcal{V}$ is a 90 neighbors scan window for each local spatial evaluation. 


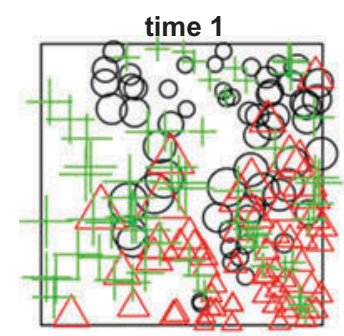

time 4

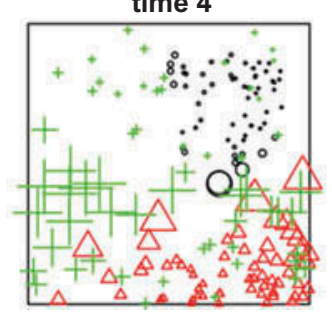

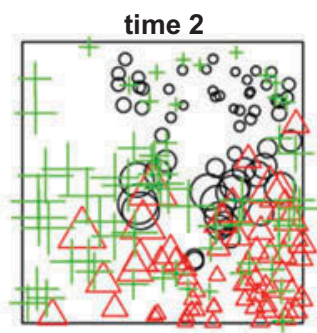

time 5

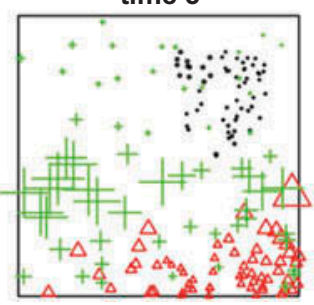

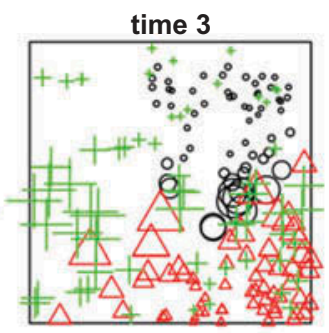

time 6

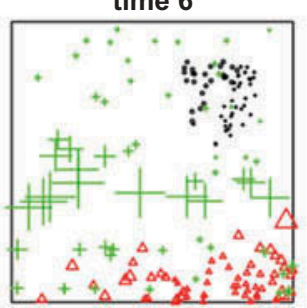

Figure 10. Local evaluations for the self- $k$-spatial entropy with $\mathcal{V}$ is a 90 neighbors scan window for each local spatial evaluation with collocation distance 0.3 .

Okabe et al. (2010)'s approach where within homogeneity assumption the local observed counts are compared to a binomial valued distribution of the expected counts. The indices based on the distance-ratios also have an internal control as the statistics do not rely on outer estimations or external hypothesis, nonetheless as seen in Figure 8 its discriminant power increases with the size of the local neighborhood used.

\section{Spatio-temporal integrated approach}

Extending a spatial analysis methodology to the spatio-temporal domain may not be as straightforward as it could seem and the literature in spatio-temporal clustering, trajectory analysis, spatio-temporal GIS is very active (Rinzivillo et al. 2008, Kuhn 2012, Bivand et al. 2013, Gabriel et al. 2013). In a previous work the spatial index given in Equation (3) has been extended to a spatio-temporal index as follows (Claramunt 2012):

$$
H_{S T}(C)=-\Sigma_{c} \frac{d_{c}^{\text {STint }}}{d_{c}^{\text {SText }}} p_{c} \log \left(p_{c}\right)
$$

where the intra-distances (respectively, extra-distances) take into account the spatiotemporal dimension, and is modeled as the product of the intra-distances (respectively, extra-distances) in space and time:

$$
d_{c}^{\text {STint }}=d_{c}^{\text {Tint }} d_{c}^{\text {Sint }}
$$

A similar multiplicative approach has been taken by Knox (1964) and particularly Mantel (1967) who built a statistic to test for spatio-temporal clustering based on the following statistic:

$$
d_{c}^{\text {STint }}=Z=1 /\left(2 * n_{\{c, c\}}\right) \Sigma_{r \in c, r^{\prime} \in c} d_{r r^{\prime}}^{T} d_{r r^{\prime}}^{S}
$$


which used standardized distance values (centered and divided by standard deviations of the distances in space separately in time and space). Two different spatio-temporal entropy indices (10) according either to using the macro-spatio-temporal distance model (11) or the Knox-Mantel or KM spatio-temporal distance model (12) can be derived and the same for the spatio-temporal versions of $(5,6,7)$.

Looking at co-occurrences and in reference to the Knox approach, the distances in (12) are now co-occurrence functions of order 2 here: $o_{r r^{\prime}}^{T}(d)=1$ if $d_{r r^{\prime}}^{T} \leq d$, with $d$ the collocation distance (called the critical time distance by Knox) and idem for space, then leading (instead of $d_{c}^{\text {STint }}$ ) to a co-occurrence spatio-temporal model $o_{c}^{\text {STint }}$ :

$$
o_{c}^{S T i n t}(d S, d T)=1 / 2 \Sigma_{r \in c, r^{\prime} \in c} o_{r r^{\prime}}^{T}(d T) o_{r r^{\prime}}^{S}(d S)
$$

counting the number of pairs of observations from the same class that are co-occurrent in space and in time, and a similar definition for $o_{c}^{S T e x t}$. A similar co-occurrence approach with the model as in (11) will still build a suitable spatio-temporal compactness ratio. The Knox approach translates directly for the co-occurrence-based indices of Section 2.1 by using as in (13) the collocation distances $d T$ and $d S$; indices have a superscript $o$, e.g., $H_{k S T}^{o}(C)$. For the other distance-ratios-based indices expressed in Section 2.2 with their spatio-temporal versions, alike (10) and with (11) or (12), the Knox approach using only co-occurrent counts instead of distance averages makes these indices more local as the collocation distances in time and space parameterize them. Nonetheless, as distances and co-occurrences are 'negatively correlated', the inverse of the ratio has to be considered in (4) and (8) as was done in (5). These distance-ratios indices, termed with also the added superscript $o$, e.g., $H_{S T}^{o}(C)$, combine the discriminant-ratios approach and the co-occurrence approach (here of order 2). For them the model expressed by Equation (13) is in fact equivalent to count the co-occurrences of pairs within a spatio-temporal vicinity similar to a 'cylinder' selection (the high of the cylinder being the collocation distance in time) before computing the ratios.

Local constraints and local evaluation as in the previous section can be combined with these type of discriminant-ratio indices. Table 1 illustrates some results for the simulated data; as it might be difficult to assess the results for all times (except with the normalized index in column three), a sliding window selection has been applied with comparison to all times.

In Figure 11, the global evaluation for the self- $k$-spatial entropy $H_{k S T}^{s o}(C, d S, d T)$ of $80.5 \%$ has been locally evaluated at time 3 and 4 , and shows a clear visual spatio-

Table 1. Global spatio-temporal entropy for different time selection. (the equation numbers involved for the indices are mentioned).

\begin{tabular}{lccc}
$\begin{array}{l}\text { Timelindex } \\
\text { value }(\% \text { of } \\
\text { all times\}) }\end{array}$ & $\begin{array}{c}H_{S T}^{\text {macro }}(C) \\
(10) \text { and }(11)\end{array}$ & $\begin{array}{c}H_{S T}^{K M}(C) \\
(10) \text { and }(12)\end{array}$ & $\begin{array}{c}H_{d S T}^{o}(C, d T, d S) \\
(5) \text { and }(13)^{*}\end{array}$ \\
\hline $\begin{array}{l}\text { time 1, time 2, time 3\} } \\
\text { time 2, time 3, time 4\} }\end{array}$ & $1.19756(113 \%)$ & $1.20049(111 \%)$ & $92.9 \%(107 \%)$ \\
time 3, time 4, time 5\} & $1.08373(103 \%)$ & $1.08513(100 \%)$ & $88.2 \%(101 \%)$ \\
time 4, time 5, time 6\} & $0.98823(93.2 \%)$ & $0.98804(91.4 \%)$ & $83.7 \%(96.2 \%)$ \\
time 1, time 3, time 5\} & $1.11353(86.1 \%)$ & $0.91298(84.5 \%)$ & $81.0 \%(93.0 \%)$ \\
all times & $1.06072(105 \%)$ & $1.13269(104.8 \%)$ & $88.4 \%(102 \%)$ \\
\hline
\end{tabular}

Note: *critical collocation distances: time 1 , space 0.3 . 
temporal clustering (small values are to look for). This pattern detection is less pronounced when using the local entropy representation with $H_{d S T}^{o}(C, d T, d S \mid \mathcal{V})$ with a global value of $87 \%$ (Table 1) and with the local punctual contributions to $H_{S T}^{K M}(C)$ (where also small values are expected when clustering structures occur). Comparing Figure 11 and previous figures on local contributions and local evaluations where only the spatial component was used, the integration of the temporal dimension brings an additional variation whatever the choice of the spatio-temporal model.

Using a macro-multiplicative or micro-multiplicative approach $(K M)$, several different ways of integrating the spatial and temporal dimensions in the indices have been derived. These main approaches can lead to other combinations such as using the macro-model with co-occurrence-based indices by performing the Hadamard product (Styan 1973) of the spatial and temporal co-occurrence tables as the observed spatio-temporal co-occurrences. Combining a local constraining and a macro or $K M$ space-time model brings another four indices based on distance-ratios purely, and two others combining distanceratios and co-occurrences when using the Knox approach, as with $H_{S T}^{o}(C)$ previously. Finally time and space can be integrated using the above series of models and applications for the entropy approaches with co-occurrences or distance-ratios but now with a nonseparate approach. This concerns local evaluations or local constraints but also the Knox approach in (13). So far being found close in space had no influence of being found close in time but it seems usually more meaningful to look further in time when locations are close in space and vice versa. This non-separable approach means for example that the spatio-temporal cylinder neighborhood around a point becomes more biconic as the spatial diskal section becomes smaller as one goes further apart in time similarly to an outbreak model (Tango 2010).

Altogether and depending on the use of global, locally constrained or local indices, using the spatio-temporal entropy indices presented in the paper means choosing two to

contributions time 3

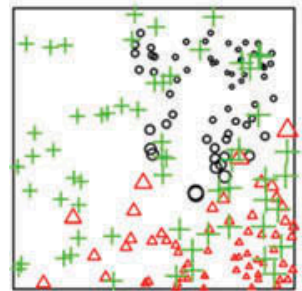

contributions time 4

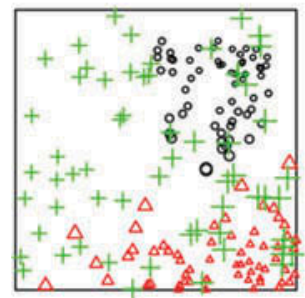

HdST time 3

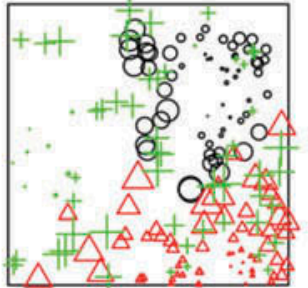

HdST time 4

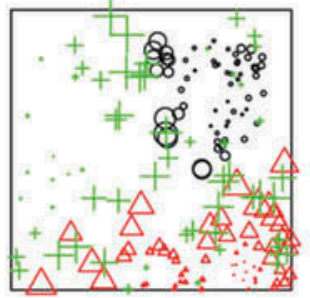

HkST time 3

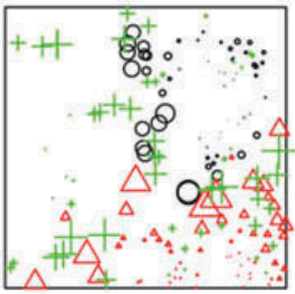

HkST time 4

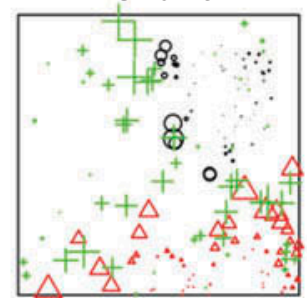

Figure 11. Local contributions to the $H_{S T}^{K M}(C)$ (left), local evaluations of the self- $k$-spatial entropy $H_{k S T}^{s o}(C, d S, d T \mid V)$ (right) and of the discriminant-ratio spatial entropy $H_{d S T}^{o}(C, d T, d S \mid V)$ (middle) at time 3 and time 4 (over the whole spatio-temporal domain with collocation distances 1 in time and 0.3 in space): local evaluations defined by $\mathcal{V}$ the 90 nearest neighbors in space and distance 1 in time. 
four parameters ( 2 for collocation, 2 for neighborhoods) but none for distance-ratios entropy as global index unless a local constraint is used. Applying a local constraint is similar to performing a local evaluation but accumulating the results for a global index. Fixing the values chosen for these parameters should be driven by the case study itself and the application requirements attached to it. Besides sample size issues when choosing for example a spatial collocation distance too small, exploring a range of parameters is part of the methodology in order to be able to describe scale variations aspects. Sensitivity analyses may be useful when reporting a specific range of parameters linked to a particular interpretation.

\section{Case study}

The application of the entropy indices on the simulated data illustrates their potential. Further experiments have been applied to a research currently under development and oriented to the study of the spatio-temporal distribution of maritime activities taking place in the Bay of Brest in North West France during the year 2009 (Le Guyader and Gourmelon 2013). This research takes place in the context of coastal seas, in which diverse activities take place, this generating an increasing pressure on the environment and often conflicting interactions (Young et al. 2007). Understanding these interactions remains a challenge for research (Leslie and McLeod 2007).

The identification of activity conflicts at sea can be modeled by superimposing activity zones (Brody et al. 2006, Beck et al. 2009, Stelzenmüller et al. 2013), and quantified using several measures of spatial intersections such as the cumulative number of activities, activity density per unit of area, presence/absence of potential conflicts or degree of potential conflict. However, the temporal dynamics of these activities are not considered, as well as it is not straightforward to qualify the way these activities interact in space and time. We formulate two hypotheses: (1) activities potentially interacting are considered in spatio-temporal interaction; (2) spatio-temporal interactions are approximated by their intersections in space and time.

Daily human activity patterns are recorded over a period of one year in 2009, and by their spatial, temporal, quantitative and qualitative properties. Data are collected from Automatic Identification System (AIS) that track maritime trajectories, and semi-structured interviews realized with stakeholders. Overall 29 activities have been recorded (e.g., fishing, water sports, maritime transportation) with a daily temporal granularity. Daily spatio-temporal intersections have been discretized with a uniform (hexagonal) lattice and aggregated monthly. For the purpose of our study spatio-temporal interactions between maritime transportation activities (labeled C111) and leisure have been considered, that is,

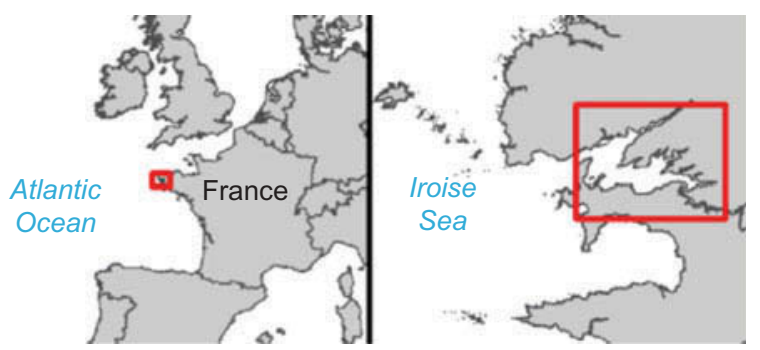


kayak (E115), windsurf (E113) and sailing (E111), this generating six classes of conflict in space and time: (E111_C111), (E111_E113), (E111_E115), (E113_C111), (E113_E115) and (E115_C111). Each spatio-temporal conflict is approximated in space by the centroid of the interaction polygons that model the intersection of the polygons where each activity takes place.

A first visual exploration (Figure 12) shows that the classes appear well delineated in space at any month. Therefore, the analysis is oriented to a study of spatio-temporal variations in order to explore the persistence or not of the conflicts of activities.

Among the entropy indices previously introduced the firsts results illustrates the use of $H_{S T}^{K M}(C), H_{d S T}^{o}\left(C, d T, d S \mid \mathcal{V}\right.$ and $H_{k S T}^{s o}(C, d S, d Y \mid \mathcal{V})$ for overall, local contributions and

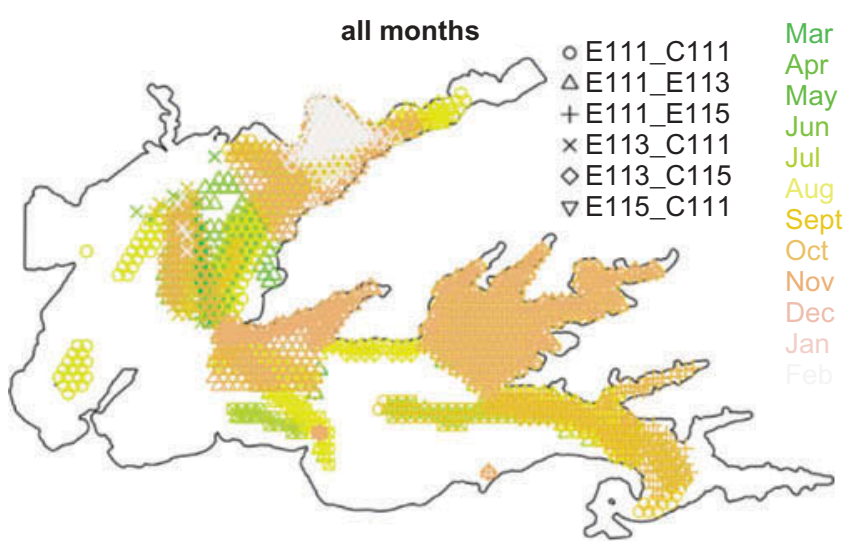

Figure 12. Visual representation of conflict classes overlapping over the months.

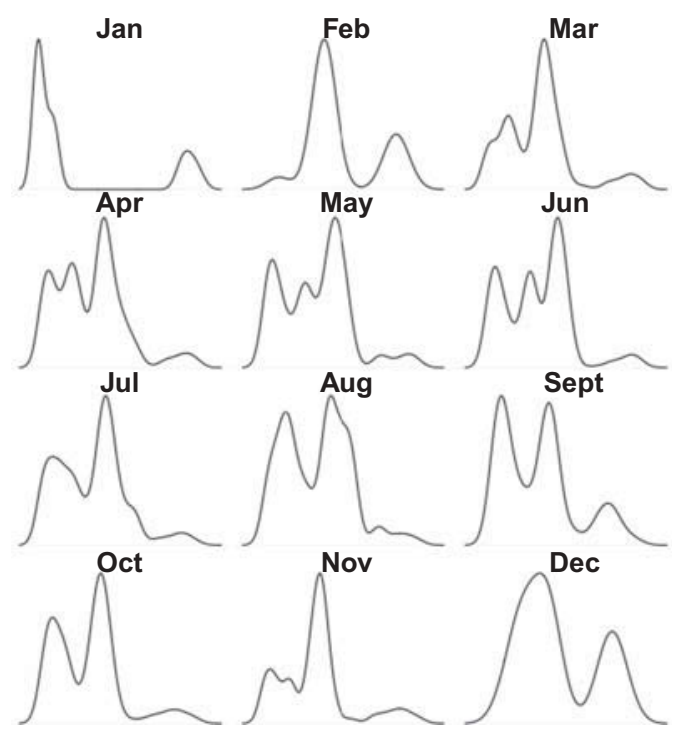

Figure 13. Densities of the local contributions to the $H_{S T}^{K M}(C)$ enabling to identify a potential spatio-temporal structure. 
local evaluations where $C$ is the categorical variable of the six classes of conflicts identified for this dataset.

Figure 13 shows that nonetheless the classes appear quite clustered in space whatever the month, they show some variations in term of spatio-temporal structuring. We focused in the neighborhood of the month of August as its density appears well structured in two comparable groups of high and low entropies (contributions to) and as being a month of high activity in general. The local evaluations allow describing and interpreting the result per classes but the indices computed locally take into account all the classes. In Figure 14 some classes like E111-C111 or E113_E115 have a tendency to have higher contributions to the spatial structure that emerges and some like E115_C111 or E113_C111 have a significant variation due to time. This observed statement from the extent and compactness of the classes observations with relatively high scores were confirmed by computing corresponding either spatial or temporal indices. Note the class E111-E113 (in red) has very homogeneous values and to a lesser extent this is also the case for class E111_E115. These two classes have shown an increase in their corresponding term in the sum making the distance-ratio-based entropy, from its computing with only spatial distances $\left(H_{s}(C)\right)$ to when using spatial and temporal distances and where the Knox-Mantel model was
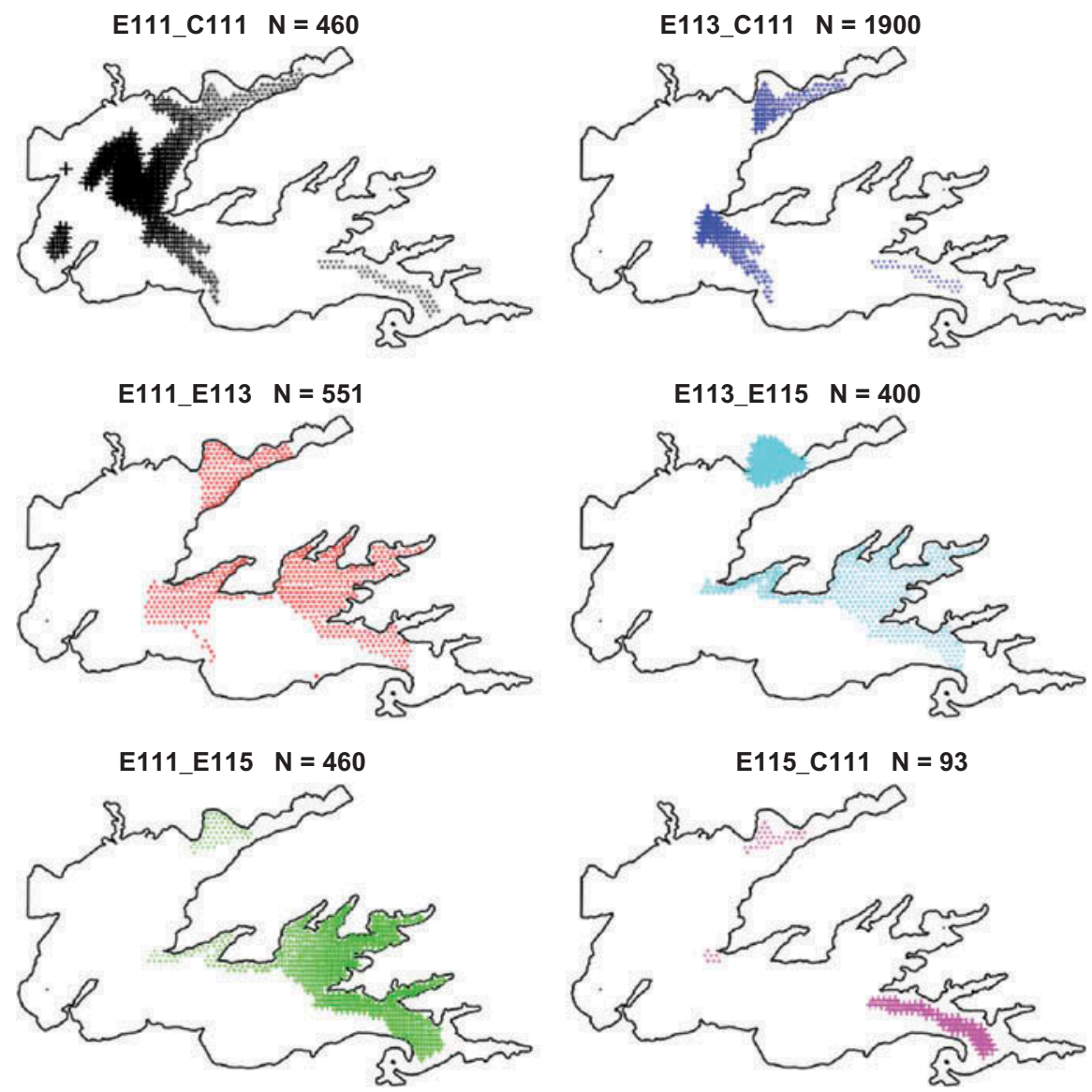

Figure 14. Local contributions at month August to the $H_{S T}^{K M}(C)$ sizes proportional to the inverse of the score (high contribution here). Split display of the six categories composing the variable $C$. 
applied $\left(H_{S T}^{K M}(C)\right)$. This expresses a lack of temporal persistence of the potential clustering for these two classes.

Figure 15 depicting local entropies for the $H_{d S T}^{o}$ index, concludes similarly to as for Figure 14 concerning the class E111_C111, nonetheless with refined clusters. The spatiotemporal structuring identified for class E113_C111 in Figure 14 are conclusive but now E111_E113 shows strong inhomogeneities. The results shown for the index $H_{k S T}^{s o}(C, d S, d Y \mid \mathcal{V})$ in Figure 16 confirm previous results altogether; the spreads of values appears more discriminative for the clustering zones previously identified, at similar locations but with different shapes. Figure 16 exhibits more evidence for clustering in areas where the logic 'cross-roads' and intense activities is verified from local knowledge.

\section{Discussion and conclusion}

Overall most of the spatial entropy indices presented in this paper and derived from a proximity concept using distances or co-occurrences, can be either mapped toward similar indices in time or extended to the spatio-temporal domain. Considering global, local and locally constrained versions of these indices, not only hints the integration of both time and space in them but in the first place gives much more flexibility to the measure of a spatio-temporal entropy.
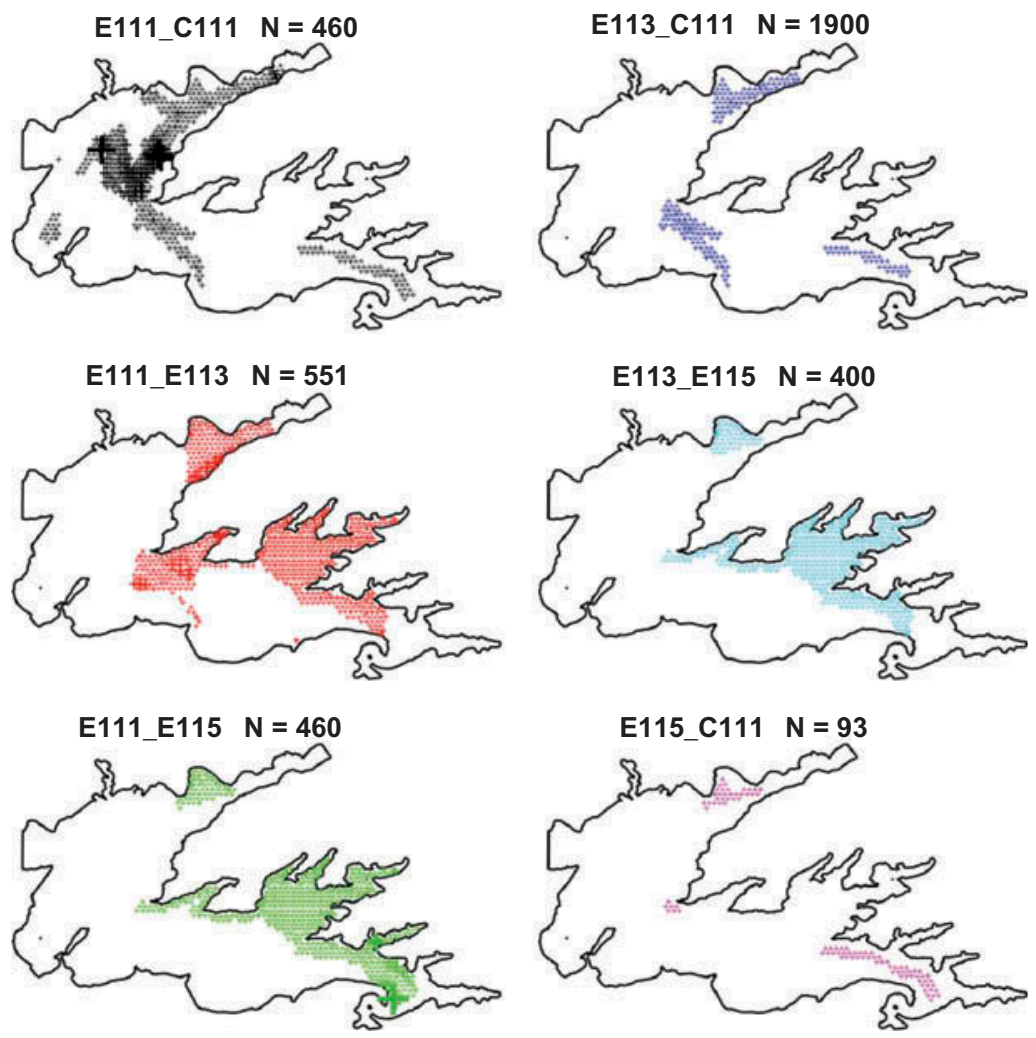

Figure 15. Local evaluations at month August of the $H_{d S T}^{o}(C, d T=330 \mathrm{~m}, d S=2$ months $\mid \mathcal{V})$ sizes proportional to the inverse of the score (high value for low entropy) and $\mathcal{V}$ is defined by the constraints $d T$ and $d S$. Split display of the six categories composing the variable $C$. 

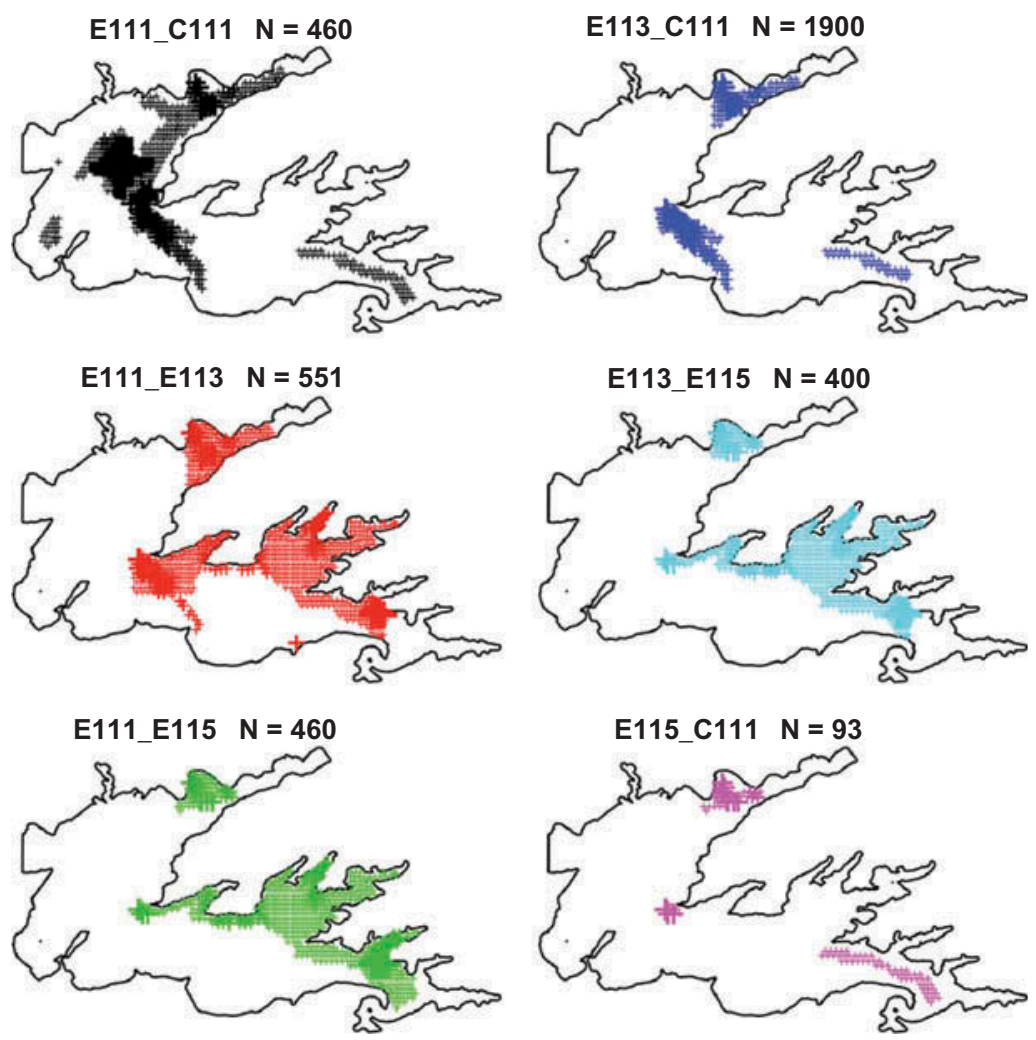

Figure 16. Local evaluations at month August of the $H_{k S T}^{s o}(C, d S=44 m, d T=1$ month $\mid V)$ sizes proportional to the inverse of the score (high value for low entropy) and is defined by 250 spatial neighbors within 2 months.

The integration of space and time in a distance-based approach can be confusing as these dimensions do not behave in the same way and scaling may not be always appropriate. Nonetheless, on the simulated data example, the macro-multiplicative and the Knox-Mantel approaches did not appear to differ much. When considering cooccurrences, the collocation distances (critical distances) allow operating in a separable way whilst still being an integrated approach but can also be thought within a space-time dependency. Besides using co-occurrences at orders $k$ greater than two, non-linear and non-homogeneous way of defining co-occurrences could be done by replacing a distance of collocation by a nearest neighbor constraint (Jacquez 1996), e.g., a co-occurrence of order 3 is counted if each of three observations is part of the $n$th nearest neighbors of each other. This is currently investigated by the authors and would allow proximity to be defined as a local concept.

Novel opportunities regarding the range of phenomena to analyze and the way of representing the results are enabled by varying the different choices and parameters, particularly the collocation distances, the neighboring and the different ratios used. Overall, the framework identified gives a high degree of flexibility to the whole approach and a potential ability of adaptation to a wide range of applications in the environmental and urban domains. In previous work the measure of spatial entropy has been already applied to the classification of agricultural and land-use data in China ( $\mathrm{Li}$ and Claramunt 
2006), in epidemiology for outbreak detection of spatial association of risk factors (Leibovici et al. 2011a), in ecology for plant communities characterizations (Leibovici et al. 2011b) and in census data population dynamics (Leibovici and Birkin 2013) where areal data was considered. Nonetheless most of these examples were mainly focusing on global assessments and were dealing with time in a non-integrated way. Post-hoc analyses, such as Monte Carlo envelopes that are applicable here, were used in order to assess the significance of the results, bringing together the structuring and clustering points of view. Computationally, distance-ratios are quite efficient as being of order two but co-occurrences of order three used here may increase computational costs for large sample sizes (spatially and/or temporally; typical run with co-occurrences was quasi instantaneous for the simulated data $-180 \times 6$, but was $3 \mathrm{mn}$ for the maritime conflict data $-2554 \times 12$ ). The functions used in this paper are currently in the process of being packaged as an $R$ package, with more computational costs results.

The spatio-temporal integration along with local exploration (local contributions, local evaluations and local constraints) as proposed in this paper offer now much more possibilities to apprehend spatio-temporal phenomena measured using an entropy framework. We were able to illustrate some of these possibilities with a simulated data and for the analysis of conflicts of activities in a maritime region in a coastal and local region. From the distributions of the occurrences and co-occurrences of actual and potential economic, social, touristic and transportation activities in a given maritime region under pressure and using the approach described in this paper enable analyzing the different interactions that arise in space and time. Amongst the dimensions to explore, the analysis of the conflicts at different scales in space, evolution of the patterns that emerge at different periods of the year as well as the respective influence of the spatial and temporal dimensions are the main aspects to explore. The first results described in the last section of this paper confirmed the potential of the different indices toward the analysis of different spatio-temporal patterns. In this example, where the classes showed visually great spatial clustering in the first place, the temporal persistence of subclusters was detected. This allows identifying much smaller clusters enabling better management and understanding of the functioning of the Bay of Brest.

\section{References}

Anselin, L., 1995. Local indicators of spatial association-LISA. Geographical Analysis, 27, 93-115. Batty, M., 1974. Spatial entropy. Geographical Analysis, 6, 1-31.

Batty, M., 2010. Space, scale, and scaling in entropy maximizing. Geographical Analysis, 42, 395421.

Beck, M., et al., 2009. Best practices for marine spatial planning. Arlington, TX: The Nature Conservancy.

Bivand, R.S., Pebesma, E.J., and Rubio, V.G., 2013. Applied spatial data: analysis with R. 2nd ed. New York: Springer.

Boots, B., 2003. Developing local measures of spatial association for categorical data. Journal of Geographical Systems, 5, 139-160.

Brody, S.D., et al., 2006. Identifying potential conflict associated with oil and gas exploration in Texas State Coastal Waters: a multicriteria spatial analysis. Environmental Management, 38 (1), 597-617.

Claramunt, C., 2005. A spatial form of diversity. In: D. Mark and A. Cohn, eds. Conference on spatial information theory (COSIT '05). Ellicottville, NY: Springer, LNCS 3693, 218-231.

Claramunt, C., 2012. Towards a spatio-temporal form of entropy. In: S. Castano, et al., eds. ER Workshops 2012, Heidelberg: Springer, LNCS 7518, 221-230.

Gabriel, E., Rowlingson, B.S., and Diggle, P.J., 2013. STPP: an R package for plotting, simulating and analyzing spatio-temporal point patterns. Journal of Statistical Software, 53, 1-29. 
Getis, A. and Ord, K., 1992. The analysis of spatial association by use of distance statistics. Geographical Analysis, 24, 189-206.

Gonzalez, A. and Chaneton, E., 2002. Heterotroph species extinction, abundance and biomass dynamics in an experimentally fragmented microecosystem. Journal of Animal Ecology, 71, 594-602.

Haining, R.P., 2003. Spatial data analysis: theory and practice. Cambridge: Cambridge University Press.

Hurlbert, S.H., 1971. The non concept of species diversity: a critique and alternative parameters. Ecology, 52, 577-586.

Jacquez, G.M., 1996. A K nearest neighbour test for space-time interaction. Statistics in Medicine, 15 (18), 1935-1949.

Jung, I., Kulldorff, M., Richard, O.J., 2010. A spatial scan statistic for multinomial data. Statistics in Medicine, 29, 1910-1918.

Karlström, A. and Ceccato, V., 2002. A new information theoretical measure of global and local spatial association: S. The Review of Regional Research, 22, 13-40.

Knox, G., 1964. The detection of space-time interactions. Applied Statistics, 13, 25-29.

Kuhn, W., 2012. Core concepts of spatial information for transdisciplinary research. International Journal of Geographical Information Science, 26, 2267-2276.

Kulldorff, M. and Nagarwalla, N., 1995. Spatial disease clusters: detection and inference. Statistics in Medicine, 14, 799-810.

Le Guyader, D. and Gourmelon, F., 2013. Modelling of human activities in coastal seas. In: 11th international symposium for GIS and computer cartography for coastal zones management, 18-21 June 2013, Victoria, BC.

Leibovici, D.G., 2009. Defining spatial entropy from multivariate distributions of co-occurrences. In: K. Hornsby, et al., eds. Spatial information theory conference on spatial information theory (COSIT '09). Vol. 5756/2009 of LNCS. Berlin: Springer, 392-404.

Leibovici, D.G., et al., 2011a. Spatially Clustered Associations in Health related geospatial data. Transactions in GIS, 15 (3), 347-364.

Leibovici, D.G., Bastin, L., and Jackson, M., 2011b. Higher-order co-occurrences for exploratory point pattern analysis and decision tree clustering on spatial data. Computers \& Geosciences, 37 (3), 382-389.

Leibovici, D.G. and Birkin, M.H., 2013. Entropic variations of urban dynamics: geocomputational perspectives. Geographical Analysis, under revision.

Leslie, H.M. and McLeod, K.L., 2007. Confronting the challenges of implementing marine ecosystem-based management. Frontiers in Ecology and the Environment, 5 (10), 540-548.

Li, H. and Reynolds, J.F., 1993. A new contagion index to quantify spatial patterns of landscapes. Landscape Ecology, 8, 155-162.

Li, X. and Claramunt, C., 2006. A spatial-based decision tree for classification of geographical information. Transactions in GIS, 10 (3), 451-467.

Li, Z. and Huang, P., 2002. Quantitative measures for spatial information of maps. International Journal of Geographical Information Science, 16, 699-709.

López, F., et al., 2010. A non-parametric spatial independence test using symbolic entropy. Regional Science and Urban Economics, 40, 106-115.

Mantel, N., 1967. The detection of disease clustering and a generalized regression approach. Cancer Research, 27, 209-220.

Margalef, R., 1958. Information theory in ecology. General Systems, 3, 36-71.

Matilla-García, M., Ruiz, J.R., and Marín, M.R., 2012. Detecting the order of spatial dependence via symbolic analysis. International Journal of Geographical Information Science, 26, 1015-1029.

McGarigal, K. and Marks, B.J., 1994. FRAGSTATS: spatial pattern analysis program for quantifying landscape structure. General Technical Report PNW-GTR-351, USDA Forest Service, Pacific Northwest Research Station, Portland, OR.

McIntosh, R.P., 1967. An index of diversity and the relation of certain concepts to diversity. Ecology, 48, 392-404.

Menhinick, E.F., 1964. A comparison of some species individuals diversity indices applied to samples of field insects. Ecology, 45, 859-861.

Okabe, A., Boots, B., and Satoh, T., 2010. A class of local and global K functions and their exact statistical methods. In: L. Anselin and S.J. Rey, eds. Perspectives on spatial data analysis, series: advances in spatial analysis. Berlin: Springer, 101-112. 
O’Neill, R.V., et al., 1988. Indices of landscape pattern. Landscape Ecology, 1, 153-162.

Openshaw, S., et al., 1987. A mark I Geographical Analysis Machine for the automated analysis of point data sets. International Journal of Geographical Information Systems, 1, 335-358.

Reardon, S.F. and O’Sullivan, D., 2004. Measures of spatial segregation. Sociological Methodology, 34, 121-162.

Riitters, K.H., et al., 1996. A note on contagion indices for landscape analysis. Landscape Ecology, 11 (4), 197-202.

Rinzivillo, S., et al., 2008. Visually driven analysis of movement data by progressive clustering. Information Visualization, 7, 225-239.

Ripley, B.D., 1977. Modelling spatial patterns. Journal of the Royal Statistical Society, 39, $172-212$.

Shannon, C.E., 1948. A mathematical theory of communication. The Bell System Technical Journal, 27, 379-423, 623-656.

Shannon, C.E. and Weaver, W., 1949. The mathematical theory of communication. Urbana, IL: University of Illinois Press.

Stelzenmüller, V., et al., 2013. Practical tools to support marine spatial planning: a review and some prototype tools. Marine Policy, 38 (1), 214-227.

Styan, G.P.H., 1973. Hadamard products and multivariate statistical analysis. Linear Algebra and its Applications, 6, 217-240.

Tango, T., 2010. Statistical methods for disease clustering. New York: Springer.

Wong, D.W.S., 2002. Modeling local segregation: a spatial interaction approach. Geographical and Environmental Modelling, 6 (1), 81-97.

Young, O.R., et al., 2007. Solving the crisis in ocean governance: place-based management of marine ecosystems. Environment, 49 (4), 20-32. 